\title{
Network Topology of Foreign Exchange Rate
}

\author{
Shamshuritawati Sharif ${ }^{1,2}$, Nur Syahidah Yusoff ${ }^{1,3}$ \& Maman A. Djauhari ${ }^{1}$ \\ ${ }^{1}$ Faculty of Science, Universiti Teknologi Malaysia, Skudai, Johor, Malaysia \\ ${ }^{2}$ College Arts and Science, Universiti Utara Malaysia, Sintok, Kedah, Malaysia \\ ${ }^{3}$ Faculty of Industrial Sciences \& Technology, Universiti Malaysia Pahang, Gambang, Pahang, Malaysia \\ Correspondence: Shamshuritawati Sharif, Faculty of Science, Universiti Teknologi Malaysia, Skudai, Johor, \\ Malaysia; College Arts and Science, Universiti Utara Malaysia, Sintok, Kedah, Malaysia. E-mail: \\ shamshurita@yahoo.com
}

Received: October 3, 2012

Accepted: October 23, 2012 Online Published: October 26, 2012

doi:10.5539/mas.v6n11p35

URL: http://dx.doi.org/10.5539/mas.v6n11p35

\begin{abstract}
In this paper we use a network topology approach to examine the interrelationship of 55 world currencies which is based on correlation analysis. With this point of view, those relationships can be measured by a correlation structure among currencies. To analyse that network, we filter the important information contained therein by using minimum spanning tree, and we interpret it by using a centrality measures. This topology will gives us a useful guide to understand their behaviour and to determine the dominance currency in the network as part of a complex network. The results show that HKD-Hong Kong Dollar is the most dominance currencies, followed by in order of importance; SGD-Singapore Dollar, USD-US Dollar, SAR-Saudi Riyal, and TWD-New Taiwan Dollar.
\end{abstract}

Keywords: adjacency matrix, complex system, correlation matrix, distance matrix

\section{Introduction}

A currency such as Malaysian ringgit, US dollar, British pound sterling, etc., can be used for payments transaction within country. In any country whose residents conduct business abroad or engage in financial transactions with residents in other countries, they need for an exchange of one currency to another currency, so that payments can be made in a form acceptable to foreigners. The exchange rates have direct influence on all other markets because the price of any asset is expressed in terms of a currency (Górski et al., 2008). A value of currency is expected to reflect the whole economic status of the country, and foreign exchange rate is considered to be measure of economic balance of the two countries (Mizuno et al., 2006).

Interestingly, a currency exchange rate constitutes a complex system. Their complex interrelationships are in terms of price fluctuations, and their number of currencies. Usually, those interrelationships can be represented by the correlation analysis among the logarithmic of currencies price returns. To filter the important information contained in such complex system, Mantegna introduces the use of a minimal spanning tree (MST) in 1999. Since then, MST is widely used in many areas in financial industry such as currency exchange rate; Mizuno et al. (2006), Kwapień et al. (2009), Górski et al. (2008), and Jang et al. (2011), while in stock market; Tabak et al. (2010) and Miccichè et al. (2003), and Onnela et al. (2003) in portfolio analysis.

Based on MST, a filtered network topology which contains important information can be constructed. Furthermore, the role of importance of each particular currency can be expressed by the use of centrality measure. Therefore, to illustrate the role of this measure, in this paper we conduct a study on the 55 world currency exchange rate. For that purpose, those currency will be viewed as a complex system consisting of 55 currencies as nodes connected by [55]*[55-1]/2=1485 links each of which corresponds to the correlation coefficient between two different nodes.

The remainder of the paper is structured as follows. We briefly review on MST in the next section, followed by the currency exchange rate data in Section 3. In Section 4 and Section 5, we discuss on its research methodology and research results, respectively. Later, in the last section, a concluding remark will be delivered.

\section{A Review on Minimum Spanning Tree}

Generally, the MST is a concept in graph theory (Diestel, 2010). In a connected weighted undirected graph of $n$ 
objects, the MST is a tree having $n-1$ edges that minimize the sum of edge distances.

Since it was introduced one decade ago by Mantegna and Stanley (2000), minimal spanning tree (MST) related to the correlation networks becomes an indispensible tool in networks analysis to filter the most important information. See, for example, Eom et al. (2008) for its application to filter the information contained in a correlation matrix, Tumminello et al. (2005) in general complex systems and Eom et al. (2006) in Shanghai and Shenzhen market index.

It has been used extensively, not limited in financial study, but have been explored by Krichel and Bakkalbasi (2006) who use it in sociology, Park and Yilmaz (2010) in transportation, and Situngkir and Surya (2004) in politics.

In practice, the MST that used in networks analysis is usually obtained from the two most suggested algorithms in the literature, i.e., Kruskal's algorithm and Prim's algorithm (Graham \& Hell, 1985; Mantegna, 1999; Mantegna \& Stanley, 2000). Huang et al. (2009) has concluded that Kruskal's algorithm is comparatively better to Prim's algorithm when the number of nodes is less than 100. On the other hand, when that number is larger than 100, Prim's algorithm is more advanced. It is no doubt that those algorithms play a very important role in the construction of network topology and economic taxonomy of stocks.

\section{Currency Exchange Rate Data}

We analyze time series of daily data for 55 currencies. The currencies are denoted according to ISO 4217 standard, and they can be divided into six groups according to their region (Jang et al., 2011).

The African countries include; EGP, GHS, JMD ZAR, and ZMK (5 currencies), American countries: ARS, BRL, CLP, COP, HNL, MXN, PEN, TTD, USD, and VEF (10 currencies), Asian countries; HKD, IDR, INR, JPY, KRW, LKR, MYR, PHP, PKR, SGD, THB, TWD (12 currencies), Pacific Ocean countries; AUD, FJD and NZD (3 currencies), and European countries; BGN, CAD, CHF, CYP, DKK, EUR, GBP, HUF, ISK, OK, PLN, RON, RUB, SEK, SKK, TRY (16 currencies). Finally the Middle East countries taken into account are: ILS, AED, DZD, JOD, KWD, LBP, MAD, TND and SAR ( 9 currencies). All those currencies can be viewed as complex systems.

The sampling period of the data collection is taken in daily basis from February 1, 2000 to April 28, 2011 from Sauder School of Business (2011). In general there are 5 trading days per week and about 20 trading days per month. In this analysis, Gold Ounces (XAU) is used as the base currency.

\section{Research Methodology}

In this section, we describe the technical methodologies used for the treatment of the data. Generally, network topology starts with a correlation matrix. Then, we transform it into a distance matrix (Mantegna \& Stanley, 2000). From this matrix we construct the corresponding sub-dominant ultrametric (SDU) distance matrix based on minimum spanning tree (MST). For this purpose we use Kruskal algorithm as suggested in Bondy and Murty (1976), Mantegna and Stanley (2000), and Diestel (2010). MST will then be used to simplify the original network and summarize the most important information. By using a Matlab version 7.8.0 (R2009a) and Pajek (Batagelj \& Mrvar, 2011), the analysis can be visualized into graphical representation, and it will help us to understand the complex system in a simple manner.

First, let $P_{i}$ is the $i$-th currencies under study where $i=1,2, \ldots, 55$. To construct currency networks, we calculate the logarithmic return of exchange rate $R_{i}$,

$$
R_{i}(t)=\ln P_{i}(t+1)-\ln P_{i}(t)
$$

for all $i=1,2, \ldots, 90$. Equation (1) defines a complex system among stocks in the form of stock networks. To filter the information contained therein, we construct a correlation matrix among those stocks, is a symmetric matrix of size $90 \times 90$ where the element in the $\mathrm{i}$-th row and $\mathrm{j}$-th column is,

$$
\rho_{i j}=\frac{\left\langle R_{i} R_{j}\right\rangle-\left\langle R_{i}\right\rangle\left\langle R_{j}\right\rangle}{\sqrt{\left(\left\langle R_{i}^{2}\right\rangle-\left\langle R_{i}\right\rangle^{2}\right)\left(\left\langle R_{j}^{2}\right\rangle-\left\langle R_{j}\right\rangle^{2}\right)}}
$$

representing the correlation coefficient between $i$-th and $j$-th stocks (Mantegna \& Stanley, 2000). That correlation coefficient quantifies the degree of linear relationship between $i$-th and $j$-th stocks. By definition, $\rho_{i i}=1$ for all $i$ and $\rho_{i j}$ can vary from -1 to 1 for all $i \neq j$ where, 


$$
\rho_{i j}=\left\{\begin{aligned}
1 & \text { means perfectly positive linear relationship } \\
0 & \text { means no linear relationship } \\
-1 & \text { means perfectly negative linear relationship }
\end{aligned}\right.
$$

To analyze the network, we transform the correlation matrix into a distance matrix by using the following formula (Mantegna \& Stanley, 2000).

$$
d_{i j}=\sqrt{2\left(1-\rho_{i j}\right)}
$$

This $d_{i j}$ is a distance between the $i$-th and $j$-th stocks since it satisfies the following three properties; (i) $d_{i j} \geq$ 0 and $d_{i j}=0 \Leftrightarrow X_{i}=X_{j}$, (ii) $d_{i j}=d_{j i}$, and (iii) $d_{i j} \leq d_{i k}+d_{k j}$. The first property tells us that two stocks that are perfectly correlated (either positive or negative), $\left|\rho_{i j}\right|=1$, will be represented by a single point in Euclidean space $\left(d_{i j}=0\right)$. Moreover, $0 \leq d_{i j} \leq 2$.

The second property is symmetric property; the distance between the $i$-th and $j$-th stocks is equal to the distance between the $j$-th and $i$-th stocks. In other words, the correlation between the $i$-th and $j$-th stocks is equal to the correlation between the $j$-th and $i$-th stocks $\left(\rho_{i j}=\rho_{j i} \Leftrightarrow d_{i j}=d_{j i}\right)$.

The last property is well known as triangular property. From (2), we conclude that, in general, the higher the correlation coefficient the smaller the distance.

By using Equation (3), we obtain a distance matrix $D$ of size $55 \times 55$ with $d_{i j}$ as the element in the $i$-th row and $j$-th column. It is the matrix that we analyze in the rest of the paper.

To visualize, simplify and summarize the important information contained in the network represented by $D$, we use the notion of sub-dominant ultrametric (SDU) as suggested in Mantegna and Stanley (2000). Let $\Delta$ be a matrix $55 \times 55$ with $\delta_{i j}$ as the element in $i$-th and $j$-th column. We call $\Delta$ the SDU matrix of $D$ if $\delta_{i j}$ is an ultrametric distance between the $i$-th and $j$-th currencies and $\delta_{i j} \leq d_{i j}$ for $i$ and $j$. By ultrametric distance we mean that (i) $\delta_{i j} \geq 0$ and $\delta_{i j}=0 \Leftrightarrow X_{i}=X_{j}$, (ii) $\delta_{i j}=\delta_{j i}$ for all $i$ and $j$, and (ii) $\delta_{i j} \leq \max \left\{\delta_{i k}, \delta_{k j}\right\}$ for all $i, j$ and $k=1,2, \ldots, 55$. SDU is determined by a MST of the original network (Benzecri, 1982; Tabak et al., 2010). In this paper, MST will be determined by using Kruskal algorithm (Kruskal, 1956).

To interpret the MST, we use degree centrality and closeness centrality. This measure is helpful to understand the importance and or influence of each node relative to the others (Xu et al., 2009; Abbasi \& Altmann, 2010; Monárrez-Espino \& Caballero-Hoyos, 2010). The role of those measures in details and its formula can be consult in Borgatti (1995).

\section{Results and Discussion}

MST of currency exchange rate, its centrality measure, and a summary will be presented in this section. To elaborate the MST we examine its distance matrix over $55 \times 55$ of currency exchange rate.

\subsection{Minimum Spanning Tree}

Figure 1 shows the correlation structure of 55 currencies exchange rate. The degree and direction of their inter-relationship is representing by the colour of the figure. In this case, instead of analyzing $55 * 55=3025$ correlation elements, we can filter the information into $55^{*}[55-1] / 2=1485$ correlation elements by using MST. However, to construct a MST, we need the adjacency matrix that corresponds to the MST. The element of adjacency matrix is $a_{i j}=1$ if the $i$-th and $j$-th nodes are linked and 0 otherwise. This matrix is a symmetric matrix and all diagonal elements are 0 . In Figure 2, empty cell represents 0 and colour cell 1 . Based on the adjacency matrix, we only have $55-1=54$ links to visualize their interrelationship. This is the advantages of the MST. 


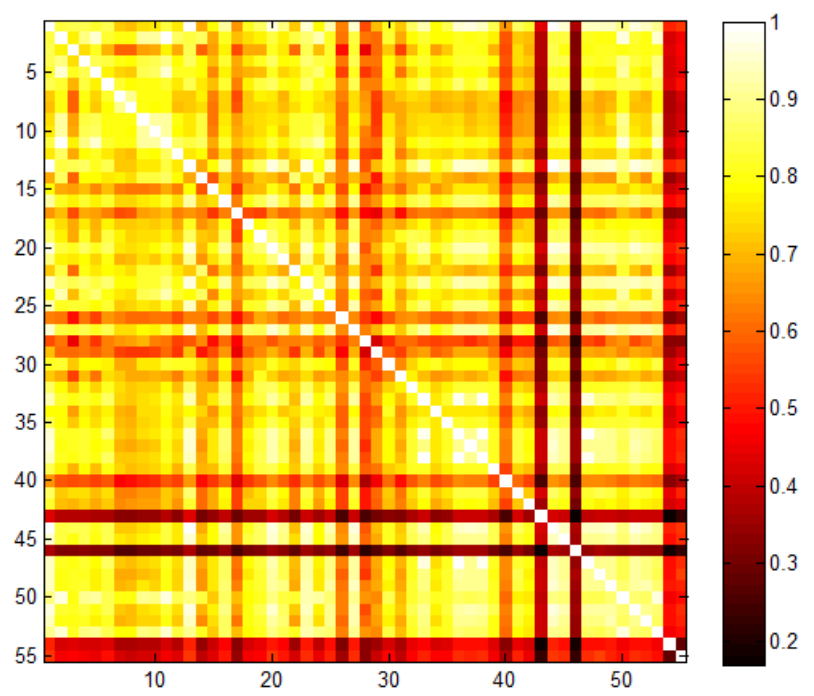

Figure 1. Correlation matrix

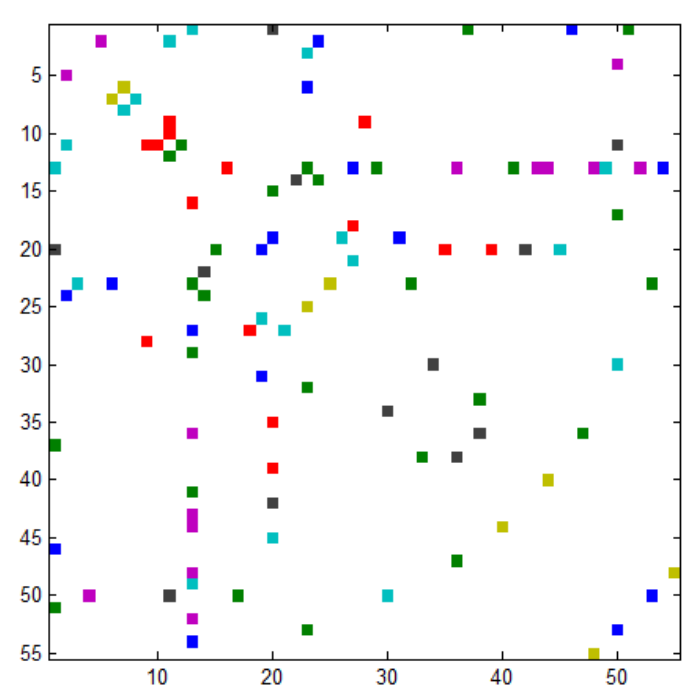

Figure 2. Dot plot matrix

The Gold base distance-matrix indicated similarity in currency dynamics between DKK-NOK (0.4071), DKK-CYP (0.5226), DKK-EUR (0.0662), and DKK-SEK (0.4091), all members of the European countries. The main surprise was the CAD which is not close to any of European countries, but it is reasonably close to SGD (0.499). In Figure 3, the link between European countries cluster (blue points) and the rest of the world is via MAD-TND-SGD-HKD.

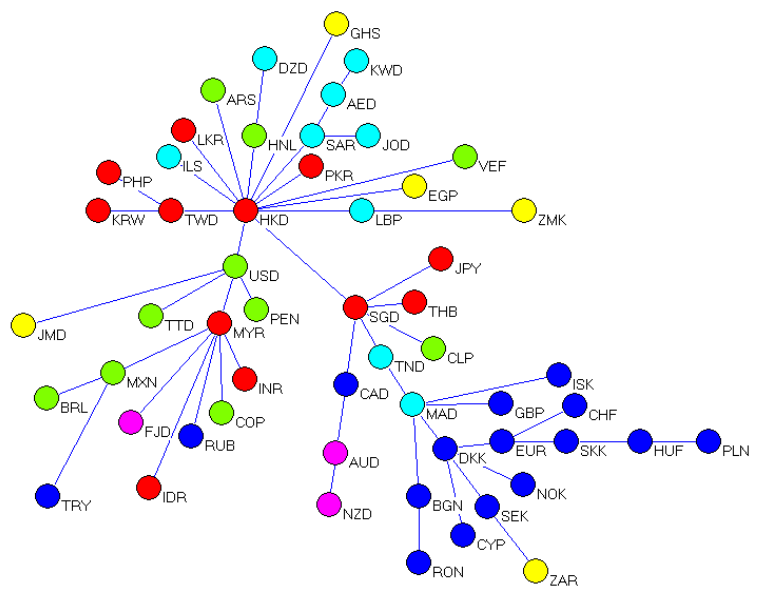

Figure 3. MST of currency exchange rate

Based on MST, it shows the currencies are clustered into four main groups which MYR, HKD, SGD, and MAD are the key currencies. The star like structure indicates the HKD is the predominant world currency, and the hub of a cluster.

In this analysis, there are 11 currencies dominated by HKD. USD is the nearest currencies to HKD (0.0536), followed by HNL, TWD, SGD, LKR, SAR, LBP, PKR, ILS, EGP, ARS, VEF, and GHS. In the other hand, the nearest currencies to USD are JMD, MYR, PEN and TTD.

INR, TWD, RUB, COP, FJD and IDR currencies are dominated by MYR. Indirectly, they will also affected by USD, since MYR is the second nearest currencies to USD. Either USD is growing up or getting loss, the changes on its will influence six of them, as well as MYR and other nodes nearby to USD node.

The other seven currencies are dominated by SGD which is THB, TND, CAD, CLP, JPY, AUD and NZD. Among all of them, JPY, CAD, AUD, and NZD are the most liquid currencies in the world (Górski et al., 2008).

Moreover, the large distance involves for USD-JMD (1.1225), HKD-GHS (1.068), HKD-VEF (0.9854), LBP-ZMK (0.9503), HKD-HNL (0.8314), and MXN-TRY (0.8056) show them to be largely isolated from the 
others. Three of them represent African countries. As a conclusion, from Figure 3, we can see that African countries, American countries, Asian countries, Pacific Ocean countries, and Middle East countries are poorly cluster, since their interrelationship are more disperse (see Figure 2). In what follow, we present the centrality measure to interpret the MST more clearly.

\subsection{Centrality Measure}

A substantial part of network theory is devoted to the idea of the centrality of a node (Newman, 2010), or how influential the node is in the network. In what follows, we perform a degree centrality and closeness centrality.

Degree centrality is the simplest centrality measure by using the local structure around nodes only. It provides information on how many other nodes are connected with a particular node. In Table 1 we present the value of measure of each node.

Table 1. Degree centrality

\begin{tabular}{llllll}
\hline Currency & & Currency & \multicolumn{3}{c}{ Currency } \\
\hline AED & 2 & HUF & 2 & PKR & 1 \\
ARS & 1 & IDR & 1 & PLN & 1 \\
AUD & 2 & ILS & 1 & RON & 1 \\
BGN & 2 & INR & 1 & RUB & 1 \\
BRL & 1 & ISK & 1 & SAR & 3 \\
CAD & 2 & JMD & 1 & SEK & 2 \\
CHF & 1 & JOD & 1 & SGD & 6 \\
CLP & 1 & JPY & 1 & SKK & 2 \\
COP & 1 & KRW & 1 & THB & 1 \\
CYP & 1 & KWD & 1 & TND & 2 \\
DKK & 5 & LBP & 2 & TRY & 1 \\
DZD & 1 & LKR & 1 & TTD & 1 \\
EGP & 1 & MAD & 5 & TWD & 3 \\
EUR & 3 & MXN & 3 & USD & 5 \\
FJD & 1 & MYR & 7 & VEF & 1 \\
GBP & 1 & NOK & 1 & ZAR & 1 \\
GHS & 1 & NZD & 1 & ZMK & 1 \\
HKD & 13 & PEN & 1 & & \\
HNL & 2 & PHP & 1 & & \\
\hline & & & & &
\end{tabular}

To visualize the result in Table 1, Figure 4 we present the MST where the size and colour of the node represent the score of centrality measure and the rank of importance, respectively. 


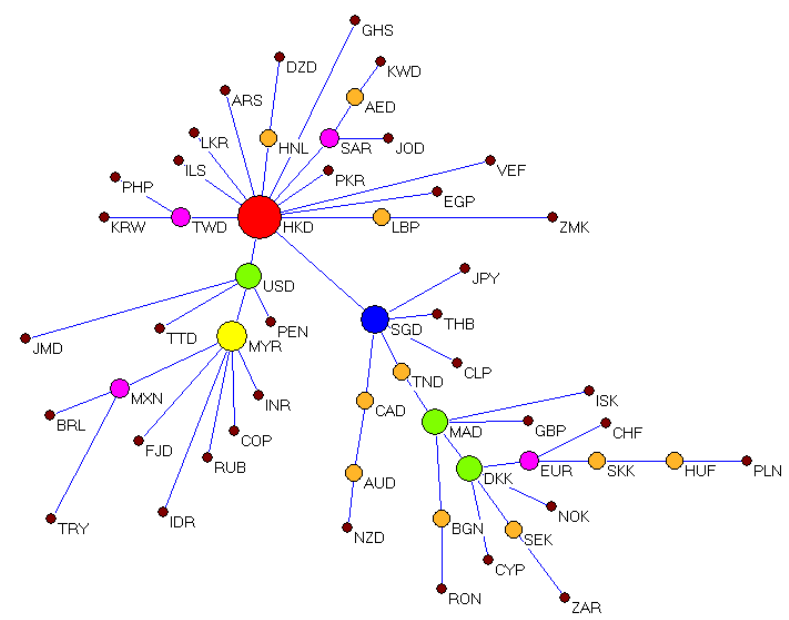

Figure 4. Degree centrality

Based on degree centrality, see Figure 4, HKD (red points) have the highest number of links (13 links) in the network followed by, in order of importance: MYR has 7 links (yellow points), and SGD has 6 links (blue points). Each of the followings has 5 and 3 links: USD, DKK and MAD (green points), and EUR, MXN, TWD, and SAR (purple points), respectively. The rests are of 1 and 2 links only. The higher the number of links is the higher the influence of a particular currency. In this analysis, HKD is the most influential currencies to the larger number of other currencies.

On the other hand, closeness centrality measures how close a node is to all other nodes in terms of correlations (Okomato, 2009). Closeness centrality can also be regarded as a measure of how long the information is to spread from a given node to other reachable nodes. Closeness centrality of node $i, c_{i}$, is the ratio of the number of links in the MST, which is equal to $(n-1)$, and the number of links in the path from $i$ to $j$ for all $j \neq i$. In Table 2 , we demonstrate the closeness centrality measure to summarize the information contained in MST.

Table 2. Closeness centrality

\begin{tabular}{llllll}
\hline Currency & \multicolumn{3}{c}{ Currency } & \multicolumn{3}{c}{ Currency } \\
\hline AED & 0.213 & HUF & 0.137 & PKR & 0.258 \\
ARS & 0.258 & IDR & 0.196 & PLN & 0.121 \\
AUD & 0.204 & ILS & 0.258 & RON & 0.170 \\
BGN & 0.205 & INR & 0.196 & RUB & 0.196 \\
BRL & 0.167 & ISK & 0.203 & SAR & 0.266 \\
CAD & 0.252 & JMD & 0.227 & SEK & 0.181 \\
CHF & 0.156 & JOD & 0.211 & SGD & 0.327 \\
CLP & 0.248 & JPY & 0.248 & SKK & 0.158 \\
COP & 0.196 & KRW & 0.209 & THB & 0.248 \\
CYP & 0.179 & KWD & 0.176 & TND & 0.287 \\
DKK & 0.218 & LBP & 0.261 & TRY & 0.167 \\
DZD & 0.208 & LKR & 0.258 & TTD & 0.227 \\
EGP & 0.258 & MAD & 0.254 & TWD & 0.263 \\
EUR & 0.184 & MXN & 0.199 & USD & 0.292 \\
FJD & 0.196 & MYR & 0.243 & VEF & 0.258 \\
GBP & 0.203 & NOK & 0.179 & ZAR & 0.153 \\
GHS & 0.258 & NZD & 0.170 & ZMK & 0.208 \\
HKD & 0.346 & PEN & 0.227 & & \\
HNL & 0.261 & PHP & 0.209 & & \\
\hline & & & & &
\end{tabular}


To visualize the result in Table 2, Figure 5 we present the MST where the size and colour of the node represent the score of centrality measure and the rank of importance, respectively.

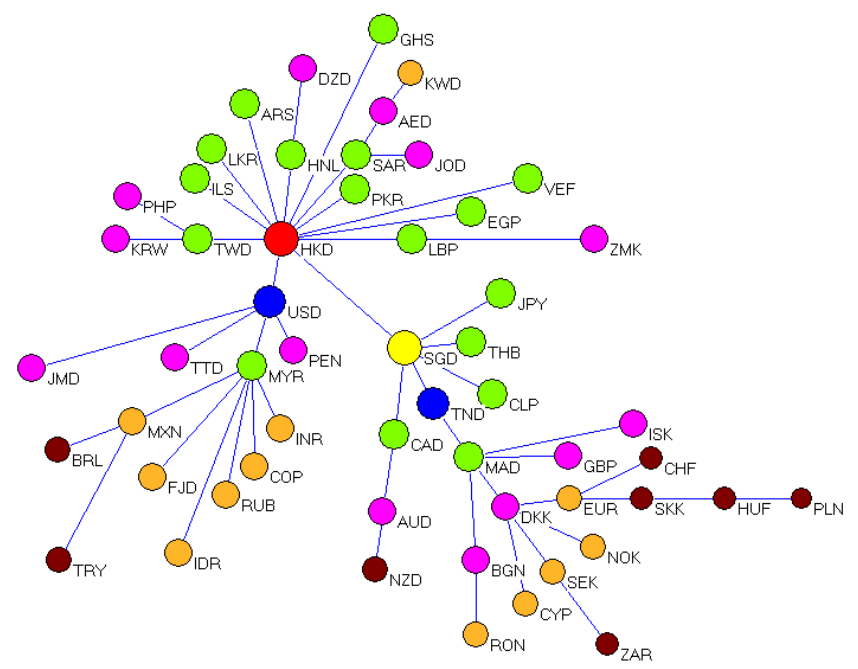

Figure 5. Closeness centrality

According to closeness centrality, see Figure 5, HKD (red points) have the highest score (0.346) in the network. HKD plays the most important role in the network followed by, in order of importance: SGD has a score of 0.327 (yellow points or the second most important), USD and TND (blue point or the third most important). Each of the followings are the fourth most important (green points): SAR, TWD, HNL, LBP, ILS, ARS, EGP, GHS, LKR, PKR, VEF, MAD, CAD, JPY, THB, CLP, and MYR. This means that those currencies strongly influence the others.

\section{Summary}

A dot plot matrix analysis (Figure 2) shows that managing the currencies exchange rate is difficult. The currencies are highly correlated within factors but not between them. According to Figure 3, we find out that the currencies are not clearly clustered from each other except for European countries, and the African countries are isolated from the others. The behaviors of clusters are not only formed according to geographical factors, but economical factors as well (Kwapien et al., 2009). In addition, the figure obviously shows that HKD is the hub of the cluster.

Based on degree centrality and closeness centrality, we perform a Pareto analysis based on the ten currencies of highest scores in each centrality measures, see Table 1 and Table 2. The following 15 currencies are the vital few in influencing foreign exchange rate; HKD, SGD, USD, SAR, TWD, MYR, TND, DKK, MAD, EUR, HNL MXN, LBP, ILS, and ARS. These six currencies should be paid more attention by the investors. If any changes in their currencies, it will influence the other currencies as well. This means that if those variables are well managed, then the others will be well influenced.

\section{Concluding Remark}

In this paper, we have introduced network topology approach to understand the correlation matrix of size $55 \times 55$. Its advantage is illustrated by using 55 currencies, and their correlation matrix is considered as a network system. To simplify that network we used MST which provides an optimal sub-network in the form of a spanning tree. This tree is then used to construct the optimal network topology of those currencies. A financial example has been presented to illustrate the advantages of this approach. One significant advantage is that network topology approach is easy to interpret since all the results can be visualized.

\section{References}

Abbasi, A., \& Altmann, J. (2010). On the Correlation between Research Performance and Social Network Analysis Measures Applied to Research Collaboration Networks. TEMEP Discussion Paper, No.2010, Seoul National University, Korea.

Batagelj, V., \& Mrvar, A. (2011). PAJEK: Program for Analysis and Visualization of Large Networks, version 2.02. Retrieved from http://pajek.imfm.si/doku.php?id=download

Benzécri, J. P. (1982). Histoire etpréhistoire de l'Analyse des Données. Paris: DUNOD. 
Bondy, J. A., \& Murty, U. R. S. (1976). Graph Theory with Applications, USA: American Elsevier Publishing Co. Inc.

Borgatti, S. P. (1995). Centrality and AIDS. Connections, 18(1), 112-114.

Diestel, R. (2010). Graph Theory (4th ed.). Heidelberg: Springer-Verlag. http://dx.doi.org/10.1007/978-3-642-14279-6

Eom, C., Oh, G., \& Kim, S. (2006). Topological properties of the minimal spanning tree in Korean and American stock markets. arXiv: physics/0612069v1 (accessed on July 22, 2010).

Eom, C., Oh, G., \& Kim, S. (2008). Statistical investigation on connected structures of stock networks in a financial time series. Journal of the Korean Physical Society, 53(96), 3837-3841. http://dx.doi.org/10.3938/jkps.53.3837

Górski, A. Z., Drożdż, S., Kwapień, J., \& Oświęcimka, P. (2008). Minimal Spanning Tree Graphs and Power Like Scaling in FOREX Networks. Acta Physica Polonica A, 114(3), 531-538.

Graham, R. L., \& Hell, P. (1985). On the history of the minimum spanning tree problem. Annals of the history of computing, 7(1), 43-57. http://dx.doi.org/10.1109/MAHC.1985.10011

Huang, F., Gao, P., \& Wang, Y. (2009). Comparison of Prim and Kruskal on Shanghai and Shenzhen 300 Index Hierarchical Structure Tree. International Conference on Web Information Systems and Mining, 237-241. http://dx.doi.org/10.1109/WISM.2009.56

Jang, W., Lee, J., \& Chang, W. (2011). Currency Crises and the Evolution of Foreign Exchange Market: Evidence from Minimum Spanning Tree. Physica A, 390, 707-718. http://dx.doi.org/10.1016/j.physa.2010.10.028

Jayawant, P., \& Glavin, K. (2009). Minimum spanning trees. Involve, 2(4), 439-450. http://dx.doi.org/10.2140/involve.2009.2.439

Krichel, T., \& Bakkalbasi, N. (2006). A Social Network Analysis in the of Research Collaboration Economics Community. Paper presented at the International Workshop on Webometrics, Informetrics and Scientometrics \& Seventh COLLNET Meeting, France.

Kruskal, J. B. (1956). On the shortest spanning subtree and the travelling salesman problem. Proceedings of the American Mathematical Society, 7(1), 48-50. http://dx.doi.org/10.1090/S0002-9939-1956-0078686-7

Kwapień, J., Gworek, S., Drożdż, S., Drozdz, S., \& Górski, A. (2009). Analysis of a network structure of the foreign currency exchange market. Journal of Economic Interaction and Coordination, 4(1), 55-72. http://dx.doi.org/10.1007/s11403-009-0047-9

Mantegna, R. N., \& Stanley, H. E. (2000). An Introduction to Econophysics: Correlations and Complexity in Finance. Cambridge UK: Cambridge University Press.

Mantegna, R. N. (1999). Hierarchical Structure in Financial Markets. European Physical Journal B, 11, 193-197. hhtp://dx.doi.org/10.1007/s100510050929

Miccichè, S., Bonanno, G., Lillo, F., \& Mantegna, R. N. (2003). Degree stability of a minimum spanning tree of price return and volatility. Physica A, 324, 66-73. http://dx.doi.org/10.1016/S0378-4371(03)00002-5

Mizuno, T., Takayasu, H., \& Takayasu, M. (2006). Correlation networks among currencies. Physica A, 364, 336-342. http://dx.doi.org/10.1016/j.physa.2005.08.079

Monárrez-Espino, J., \& Caballero-Hoyos, J. R. (2010). Stability of Centrality Measures in Social Network Analyses to Identify Long-Lasting Leaders from an Indigenous Boarding School of Northern Mexico. Estudiossobrelas Culturas Contemporaneas, 16(32), 155-171.

Newman, M. E. J. (2010). Networks: An Introduction. Oxford: Oxford University Press.

Okamoto, K., Chen, W., \& Li, X. Y. (2008). Ranking of closeness centrality for large scale social networks. In F. P. Preparata, X. Wu, \& J. Yin. (Eds), Frontiers in algorithmics, (pp. 186-195). Berlin Heidelberg: Springer-Verlag. http://dx.doi.org/10.1007/978-3-540-69311-6_21

Onnela, J. P., Chakraborti, A., Kaski, K., Kert'esz, J., \& Kanto, A. (2003). Dynamics of market correlations: Taxonomy and portfolio analysis. Physical Review E, 68, 056110(1)-056110(12). http://dx.doi.org/10.1103/PhysRevE.68.056110

Park, K., \& Yilmaz, A. (2010). A Social Network Analysis Approach to Analyze Road Networks. Paper 
presented at the ASPRS Annual Conference, San Diego, CA.

Sauder School of Business. (2011). PACIFIC Exchange Rate Service. Retrieved from http://x.sauder.ubc.ca.

Situngkir, H., \& Surya, Y. (2004). Hierarchical taxonomy in multi-party system. Working Paper WPM 2004 Bandung Fe Institute. Pre-print: arxiv: nlin.PS/0405005

Tabak, B. M., Serra, T. R., \& Cajueiro, D. O. (2010). Topological properties of stock market networks: The case of Brazil. Physica A, 389, 3240-3249. http://dx.doi.org/10.1016/j.physa.2010.04.002

Tumminello, M., Aste, T., Matteo, T. D., \& Mantegna, R. N. (2005). A tool for filtering information in complex systems. Proceedings of the National Academy of Science USA, 102, 10421-10426. http://dx.doi.org/10.1073/pnas.0500298102

Xu, Y., Ma, J., Sun, Y., Hao, J., Sun, Y., \& Zhao, Y. (2009). Using Social Network Analysis As A Strategy For E-Commerce Recommendation. Paper presented at the Pacific Asia Conference on Information Systems (PACIS), India. 